\title{
From CUR's President
}

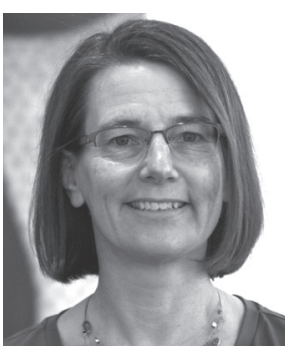

U ndergraduate research abroad. What is not to love about this idea? Two high-impact practices wrapped up in one great experience! The authors of the articles in this volume of CUR Quarterly provide compelling evidence for the value of undergraduate research abroad. As students engage in these two high-impact practices, they achieve many of the intellectual and personal gains with which we are familiar in undergraduate research community, such as ability to develop hypotheses, competency in use of tools within the discipline, proficiency in data analyses, understanding of the research process, and enhanced communication skills. On top of these outcomes, students develop intercultural competence and gain cultural awareness. Students return to their home country with a much deeper understanding of the global nature of knowledge generation and an enhanced ability to work across difference. An increased self-awareness and a higher level of comfort in taking risks also correspond with a research abroad experience. For institutions eager to combine these two high-impact practices, the articles in this volume of CUR Quarterly not only detail these outcomes but also describe successful models.

Undergraduate research abroad is one component of the growing movement in higher education to develop, enhance, and promote undergraduate research in countries across the globe. The World Congress on Undergraduate Research is another way in which undergraduate research students recently connected their work to the global community. The World Congress was hosted at Qatar University on November 12-15, 2016, and supported by CUR, the British Conference on Undergraduate Research (BCUR), and the Australasian Council for Undergraduate Research (ACUR). Collaborating across nations to present a multidisciplinary world congress, CUR contributed to a transformative undergraduate research event at which faculty and students came together from around the globe to listen and learn from one another; to share knowledge; and to discuss challenges such as climate change, sustainability, global health, information technology, and war and peace in the 21st century. Students participating in this powerful event will undoubtedly have returned to their home campuses energized and ever more ready to take on the challenges facing our world.

Although I was unable to attend this watershed event, my own thinking about undergraduate research has been significantly affected by international connections made through CUR, especially from the CUR preconference workshop at the annual meeting of the International Society for the Scholarship of Teaching and Learning. Learning about the approaches to assessment of undergraduate research in Australia, the integration of research into the curriculum in Great Britain, and the significant relationship between library faculty and undergraduate research at Canadian institutions has made me think differently about my own approaches to mentoring students and to developing undergraduate research programs. Because I have learned a great deal from these international connections, I was especially pleased that CUR hosted an institute, Initiating and Sustaining Undergraduate Research Programs, abroad this fall. Carleton University in Ottawa, Canada, hosted this institute with a number of participating U.S. and Canadian institutions, allowing for meaningful cross-country exchanges.

As undergraduate research continues to be of growing importance to higher education in nations all across the world, CUR is excited to continue dialogue with faculty from different countries and with international groups to explore ways that will enhance students' abilities to generate new knowledge and engage in global problem-solving. Our students will benefit from doing and presenting research abroad, as they see how scholarship in their discipline is conducted in different cultures and contexts, and as they experience transformative cultural exchanges. Similarly, as faculty members and administrators learn how undergraduate research is practiced elsewhere across the globe, we will gain valuable insights into how to improve our own programs so that each of us involved in undergraduate research can offer the very best educational experiences possible within our own contexts.

\section{Susan Larson}

CUR President

Concordia College, Moorhead, MN

doi: $10.18833 /$ curq/37/3/5 